\title{
Percepciones sociales frente al fenómeno de migración venezolana en una muestra de los habitantes de la ciudad de Sincelejo, Sucre, año 2019*
}

Social perceptions about the phenomenon of Venezuelan migration in a sample of the inhabitants of the city of Sincelejo, Sucre, 2019

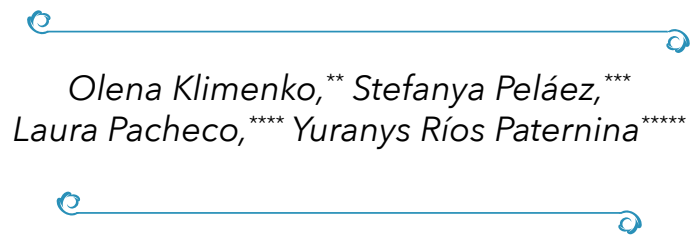

Recibido 19. 04. $2020 \bullet$ Arbitrado 15. 05. $2020 \bullet$

Aprobado 12.06. 2020

Articulo presenta resultados de la investigación llevada a cabo para optar el título de Especialista en Gestión de procesos psicosociales en la Fundación Universitaria Claretiana.

* Psicóloga, Magister en Ciencias Sociales, Doctora en Psicopedagogía, Docente Institución Universitaria de Envigado y FUCLA. ORCID: 0000-0002-8411-1263, correo: olenak45@gmail.com

*** Psicóloga, Aspirante al título de Especialista en Gestión de procesos psicosociales en la Fundación Universitaria Claretiana, stefanya.pelaez@cecar.edu.co

**** Psicóloga, Aspirante al título de Especialista en Gestión de procesos psicosociales en la Fundación Universitaria Claretiana, laura20133030@gmail.com

**** Trabajadora Social, Aspirante al título de Especialista en Gestión de procesos psicosociales en la Fundación Universitaria Claretiana,yurariosp1994@gmail.com

\section{Resumen}

Introducción: El fenómeno de migración venezolana ha producido un impacto en distintas esferas en la población colombiana, produciendo a menudo actitudes de rechazo y discriminación hacia la población migrante por parte de los habitantes nativos.

Objetivo: describir las percepciones sociales de los habitantes de la ciudad de Sincelejo frente al fenómeno de migración venezolana durante el año 2019

Método: En el estudio cuantitativo, no experimental expostfacto, de nivel descriptivo, participaron 100 habitantes de Sincelejo, la selección de la muestra se realizó de forma aleatoria mediante la encuesta realizada en las calles de la cuidad. Se empleó un cuestionario, diseñado ad hoc para el presente estudio, validado mediante el juicio de expertos y prueba piloto, obteniendo un alfa Cronbach de ,83. 
Resultados: Se observó una puntuación alta en la subescala de infidelidad, lo cual revela una postura negativa y culpabilizante al respecto de los problemas en las relaciones amorosas de los habitantes encuestados, percibida a partir de la presencia de mujeres migrantes venezolanas, siendo ésta más pronunciada en los participantes con los niveles educativos y estratos socioeconómicos más bajos. Altos puntajes en la subescala de seguridad ciudadana (mayor percepción de amenaza en los estratos socioeconómico más altos), orden público (mayor percepción de la amenaza en los estratos más bajos) y oportunidad de empleo (mayor percepción de la amenaza en los niveles educativos más altos), indican los habitantes encuestados perciben a los migrantes como una amenaza para su comunidad en estos aspectos. Igualmente, se encontraron puntajes medio altos en xenofobia, obteniendo el género masculino mayor puntajes en comparación con el femenino, al igual como participantes con un menor nivel educativo. También se encontraron niveles medio altos en el rechazo a los hábitos culturales de migrantes y tendencia de dominar por la fuerza la cultura ajena, lo cual no posibilita la integración pacifica de los migrantes a la comunidad receptora.

Conclusiones: El estudio reveló una situación bastante preocupante al respecto de una actitud altamente negativa observada en los participantes del estudio hacia los migrantes venezolanos. se recomienda seguir en esta línea de investigación en otras poblaciones con el fin de buscar estrategias de abordaje para disminuir el impacto negativo de estas precepciones sociales en la población migrante y posibilitar una mejor y más inclusiva integración de estos en las comunidades de recepción.

Palabras clave: migración venezolana, percepciones sociales, xenofobia, aculturación, hábitos de vida.

\section{Abstract}

Introduction: The phenomenon of Venezuelan migration has had an impact in different spheres on the Colombian population, often producing attitudes of rejection and discrimination towards the migrant population by the native inhabitants.

Objective: to describe the social perceptions of the inhabitants of the city of Sincelejo regarding the phenomenon of Venezuelan migration during the year 2019.

Method: it was the non-experimental, descriptive level study, with the sample of 100 habitants of Sincelejo, the selection of the sample was carried out randomly through the survey carried out in the streets of the city. Was used a questionnaire designed ad hoc for the present study, validity by expert judgment and pilot test, obtaining a Cronbach alpha of .83 .

Results: A high score was observed in the subscale of infidelity, which reveals a negative and guilty stance regarding the problems in the love relationships of the 
inhabitants surveyed, perceived from the presence of Venezuelan migrant women, being this more pronounced in the participants with the lowest educational levels and socioeconomic strata. High scores on the subscale of citizen security (higher perception of threat in the higher socioeconomic strata), public order (higher perception of threat in the lower strata) and employment opportunity (higher perception of threat in higher educational levels). high), indicate the inhabitants surveyed perceive migrants as a threat to their community in these aspects. Likewise, medium high scores were found in xenophobia, with the male gender obtaining higher scores compared to the female, as well as participants with a lower educational level. Medium high levels were also found in the rejection of the cultural habits of migrants and the tendency to dominate the foreign culture by force, which does not allow the peaceful integration of migrants to the receiving community.

Conclusions: The study revealed a very worrying situation regarding the highly negative attitude observed in the study participants towards Venezuelan migrants. It is recommended to continue in this line of research in other populations in order to search for approach strategies to reduce the negative impact of these social perceptions on the migrant population and enable a better and more inclusive integration of these in the receiving communities.

Key words: venezuelan migration, social perceptions, xenophobia, acculturation, life habits.

\section{Introducción}

La Organización Internacional para las Migraciones (OIM, 2010) define a un migrante como cualquier persona que se desplaza o se ha desplazado a través de una frontera internacional o dentro de un país, fuera de su lugar habitual de residencia independientemente de su situación jurídica, el carácter voluntario o involuntario del desplazamiento, las causas del desplazamiento o la duración de su estancia. Las causas de las migraciones son múltiples, como búsqueda de mejores condiciones de vida, violencia y guerras, desigualdades económicas y sociales, las violaciones de derechos humanos, entre otros. En la sociedad actual globalizada, los movimientos migratorios permiten a muchas personas mejorar su calidad de vida, logrando más dignas condiciones en el extranjero. Según DAES (2016), en 2015 había aproximadamente 244 millones de migrantes internacionales a nivel mundial, cifra correspondiente a 3,3\% de la población. Y OIM (2010) proyecta que para el 2050 el total mundial de migrantes internacionales llegara a 405 millones. Además, existen migraciones y desplazamiento internos, que se producen a partir de 
problemas económicos y conflictos internos de los países. En el 2016 existían 40,3 millones de desplazados internos en todo el mundo y 22,5 millones de refugiados (ACNUR, 2017).

En este orden de ideas, Venezuela es un país que se ha visto afectado por este fenómeno, debido a los altos índices de migración que datan del año 2014, causados por la difícil situación socioeconómica y política del país, sumado al aumento de criminalidad e impunidad (Ripoll et al., 2018).

Según la OIM (2018) en el año 2015 entraron regularmente a distintos países receptores 697.562 venezolanos; en el año 2017, 1.622.109 venezolanos, cifra que es superior pues no incluye a los venezolanos con otra nacionalidad, que en parte han emigrado con pasaportes extranjeros. La emigración venezolana como en los demás países, ha sido impulsada por factores determinantes macro de migración, la situación del país promueve en los venezolanos la decisión de emigrar independientemente de su nivel educativo u ocupación, tratándose de una situación migratoria generalizada que desemboca en un problema demográfico.

Colombia es el país del cono sur más afectado por el fenómeno migratorio procedente de Venezuela, las cifras presentadas por migración Colombia muestran que 1'408.055 migrantes venezolanos se encontraban en el país hasta el 30 de junio del año 2019. De estos, 742.390 son migrantes regulares y 665.665 son irregulares, asegura el informe (El Espectador, 2019).

De los diferentes enfoques para el estudio de procesos migratorios, se cuenta con un aspecto importante como es el económico, el cual acapara en los determinantes de los flujos migratorios, la integración de la población inmigrante en los países receptores y el impacto económico de la migración tanto en el país de origen, como países receptores (Bermúdez et al., 2018).

El fenómeno migratorio afecta de diferentes formas a los países receptores. Entre los efectos negativos se puede destacar tales como: aumento de la población con consecuente falta de empleo y aumento de costos de servicios sociales de educación y salud para la creciente población migrante; disponibilidad de una mano de obras más barata afectando las posibilidades de empleo para los habitantes nativos y disminución de salarios; surgimiento de diferencias raciales y culturales, que pueden desembocarse en rechazo, exclusión social o violencia explicita; entre otros (Suárez, 2008; Consejo Nacional de Población, 2010; Gonzáles, 2011).

En este orden de idas, las dificultades en la integración social de los emigrantes desembocan en las actitudes de xenofobia que afecta no solo a la población migrante, sino al tejido social de la población receptora. El estudio 
realizado por OXFAM en el año 2019 en Colombia, Ecuador y Perú, principales países receptores de la migración venezolana, muestra la presencia de opiniones cambiantes, ambivalente y contradictorias al respecto de la situación. Las personas expresan, por un lado, la simpatía y necesidad de colaboración a los emigrantes venezolanos, $\mathrm{y}$, por otro lado, temor y precaución, relacionados con distintos prejuicios. Uno de estos prejuicios es el predominante machismo en los tres países, donde mas de la mitad de la población consultada expresan que prejuicio de que las mujeres venezolanas migrantes terminará ejerciendo la prostitución. También expresan el temor sobre la precarización del empleo, el 'colapso de los servicios' y los problemas en la seguridad social, asociando la migración con la inseguridad y delincuencia (Malax et al., 2019).

Sin embargo, muchas de estas preocupaciones se refieren a las problemáticas del sistema económico y social ya preexistentes, que fueron solo exasperadas por la llegada de emigrantes. Sin embargo, en el mundo contemporáneo globalizado los medios de comunicación y las redes sociales ejercen un gran poder de influencia y manipulación de las masas, contribuyendo en la formación de percepciones sociales de las poblaciones receptoras sobre la migración y activando, a menudo, actitudes, acciones y comportamientos xenofóbicas (Velarde, 2019).

La migración en sí no es causa de problemáticas que, con frecuencia, se le atribuyen. Por esta razón es necesario que sea gestionada y comunicada de forma correcta, con el fin de que pueda contribuir a la economía de los países receptores, generando empleos y enriquecer las culturas, construyendo sociedades con diversidad que puedan convivir en paz.

En este orden de ideas, la cuidad de Sincelejo, al igual como otras partes de Colombia, ha tenido el impacto de la migración venezolana, siendo afectada en sus diferentes calles, específicamente en sitios estratégicos del centro de la ciudad, ubicándose la población de migrantes de Venezuela con empleos informales, destacando entre ellos venta de dulces, venta de mecatos en los semáforos, venta de artesanía fabricadas por los mismos, o simplemente pedir dinero, todo lo anterior con el fin de satisfacer necesidades humanas. La presencia de emigrantes ha generado impacto en los habitantes de la ciudad en cuanto a los cuestionamientos y señalamientos, a menudo negativos, como causantes de alto índice de desempleo, incluyendo migrantes profesionales que disminuyen las oportunidades en la esfera empresarial de la ciudad; invasión del espacio público y, en parte, sentimientos de lastima ante el padecimiento de múltiples necesidades en niños, niñas y adultos.

Desde el Ministerio de salud, el gobierno colombiano ha orientado acciones que permiten hacer frente a esta situación problema como la promoción de 
participación de "asociaciones de migrantes u otras formas de organización de población migrante y retornada, en los escenarios de participación del sector y otros sectores que propendan por el mejoramiento de sus condiciones de vida y mejores resultados en salud" (Ministerio y salud y proteccion social, 2014, p. 60).

A nivel local, desde la alcaldía de Sincelejo se han realizado censos a la población venezolana que dan lugar a un diagnostico que generan políticas para ayudar a dicha población y mejorar sus condiciones de vida; sin embargo, el impacto social de esta problemática ha trascendido a tal magnitud que ambas poblaciones se han visto afectadas por las secuelas de este fenómeno. En cuanto a la población migrantes, ha sido víctima de rechazo, estigmatización y/o vulneración de algunos derechos fundamentales. En cuanto a la población receptora, se pueden evidenciar rasgos de xenofobia y conductas hostiles que se convierte en una problemática de índole social con repercusión en la seguridad pública de la ciudad y en la conducta de los receptores frente a los desplazados.

Desde una mirada teórica a nivel nacional e internacional se han adelantado estudios para analizar las percepciones desde un enfoque social para entender el fenómeno de migraciones, indicando la presencia de altas cifras de conductas de agresión, discriminación, prejuicios negativos en contra de los extranjeros, vulneración de derechos y esquemas mentales que van en contra de la humanidad y los derechos fundamentales del ser humano (Lara, 2007; Cea D’Ancona, 2005).

Las concepciones o perspectiva de este fenómeno en la comunidad nativa a nivel internacional dejan ver la amenaza en la que se puede convertir la construcción de concepciones erradas o negativas en torno a la situación migratoria internacional y específicamente a nivel nacional en nuestra cercanía con Venezuela como el país con mayor flujo migratorio en las últimas décadas.

De la misma forma, las estadísticas de aumento de la población migrante también han desencadenado gran preocupación por entender otros fenómenos que podrían ser consecutivos de este, el interés por revalidar teorías y diseñar políticas públicas en prevención a acciones de violencia o violación de los derechos humanos, actualmente se convierte en una punta de lanza desde el campo psicosocial, debido a la pertinencia de abordar una realidad que incluye y afecta intereses individuales y colectivos. Siendo importante, en este aspecto, comprender más a fondo las posturas de los habitantes nativos frente al fenómeno migratorio, el presente estudio se orientó a indagar por las percepciones sociales de los habitantes de la ciudad de Sincelejo frente a la migración venezolana. 


\section{Metodología}

Diseño: estudio cuantitativo, no experimental expostfacto, con un nivel descriptivo.

Participantes: en la investigación participaron 100 habitantes de la cuidad de Sincelejo. Se realizó un muestreo aleatorio simple, mediante la técnica de abordaje al azar de personas en distintos lugares de la cuidad, con el fin de asegurar una muestra más amplia, teniendo en cuenta una población variada de distintos niveles educativos y estratos sociales, y participación de ambos sexos, con el fin de buscar una mayor diversidad de los resultados obtenidos. La muestra del estudio estuvo compuesta por 44 personas de género masculino, 56 de género femenino, de los cuales $22 \%$ son de nivel educativo básico o primaria, $23 \%$ bachilleres, $44 \%$ universitarios y $11 \%$ con educación a nivel de postrado, pertenecientes a los estratos 1 (30\%), 2 (56\%), estrato $3(10 \%)$ y estratos 4 y 5 (4\%).

Instrumentos: para el estudio se diseñó una escala Likert de 36 ítems con opciones de respuesta entre 1 y 5 , que se distribuían en siete subescalas: xenofobia, oportunidades de empleo según la nacionalidad, orden público, seguridad ciudadana, hábitos culturales, aculturación, infidelidad. Se efectúo una prueba piloto mediante la cual se ajustó la redacción de los ítems y, posteriormente, la escala fue valorada por jueces, obteniendo índice Kappa de 1 con $\mathrm{p}=, 040$. Finalmente, se realizó el cálculo de Alfa Cronbach que arrojó un valor satisfactorio de fiabilidad de escala de ,83.

Procedimiento: para la aplicación del instrumento se ejecutaron salidas en distintos lugares de la cuidad con mayor afluencia de personas, se abordaron al azar las personas para explicarles la finalidad del estudio y exponer la invitación a participar. En caso de una respuesta positiva, se firmó el consentimiento informado con cada participante y se procedió a aplicar el instrumento.

Aspectos éticos: la investigación se clasifica como un estudio de riesgo mínimo, cumpliendo con regulaciones establecidas en la Ley 1090 de 2006 del Colegio Colombiano de Psicólogos, y la Resolución 8430 de 1993 del Ministerio de Salud, que reglamentan las normas administrativas, científicas y técnicas para las investigaciones en salud.

Análisis de datos: la prueba de normalidad variables indicó la distribución no normal de todas las variables del estudio. Se empleó la estadística descriptiva para identificar las medias y desviación típica de valores de las variables de 
estudio. Se empleó el estadístico de U de Mann-Whitney para comparación de dos grupos, y el estadístico de Kruscal-Wallis par más de dos grupos.

\section{Resultados}

La puntuación obtenida mostro niveles medio alto y altos en todas en los subdimensiones de la escala.

El más alto puntaje, ubicándose en un nivel alto, se obtuvo en la variable de infidelidad (M 3,5/Dt. 1,1), seguido por seguridad ciudadana (M 3,3/Dt.0,8), orden público (M 3,2/Dt. 0,5) y oportunidad de empleo (M 3,1/ Dt.0,4), indicando una considerablemente alta actitud negativa de los participantes del estudio frente a los migrantes venezolanos en estos aspectos, considerando que el ordenamiento y el desarrollo social se han visto afectado por la llegada de los venezolanos, cuya presencia es percibida como una amenaza de seguridad para la población receptora. Uno de los aspectos relevantes, es el más alto puntaje obtenido en la variable de infidelidad, lo cual revela una postura negativa y culpabilizante al respecto de los problemas en las relaciones amorosas de los habitantes encuestados, percibida a partir de la presencia de mujeres migrantes venezolanas.

En las variables de aculturación (M 3/Dt. 1), xenofobia (M 2,6/Dt. 1) y hábitos culturales (M 2,5/Dt. 0,8), se encontró un nivel medio alto de puntuación, mostrando un considerable nivel de rechazo por las costumbres o prácticas de las personas migrantes, percepción de una desigualdad y choque cultural, donde la cultura propia debe dominar por la fuerza o la violencia a la cultura migrante; e, igualmente, un nivel muy preocupante de xenofobia, que no facilita la integración pacifica de la población migrante a la comunidad receptora.

Tabla N 1. Valores descriptivos de las variables de estudio

\begin{tabular}{lc}
\hline & $\mathrm{M}(\mathrm{Dt})$ \\
\hline Xenofobia & $2,6(1)$ \\
Oportunidad de empleo & $3,1(, 4)$ \\
Orden publico & $3,2(, 5)$ \\
Seguridad ciudadana & $3,3(, 8)$ \\
Hábitos culturales & $2,5(, 8)$ \\
Aculturación & $3(1)$ \\
Infidelidad & $3,5(1,1)$ \\
\hline
\end{tabular}

Fuente: Autores 
La comparación de variables de estudio según los grupos de género indicó la diferencia significativa estadísticamente en xenofobia a favor del género masculino, indicando que los hombres tienen una mayor tendencia a actitudes y conductas xenofóbicas, a diferencia de las mujeres.

En la variable de orden público el género femenino obtuvo la diferencia estadísticamente significativa a su favor, revelando que las mujeres perciben con una mayor amenaza la llegada de los migrantes para el equilibrio comunitario.

Y, por último, se identificó la diferencia significativa en la variable de hábitos culturales, resultado que muestra que los hombres perciben de forma más negativa las costumbres y hábitos culturales de la población migrante.

Tabla N 2. Comparación de variables de estudio según genero

\begin{tabular}{lcccc}
\hline & $\begin{array}{c}\text { Mujeres } \\
\text { Me (Ri) }\end{array}$ & $\begin{array}{c}\text { Hombres } \\
\text { Me (Ri) }\end{array}$ & U de Mann-Whitney & Valor p \\
\hline Xenofobia & $2,5(1,2)$ & $2,9(1,9)$ & 949,500 &, 049 \\
Oportunidad de empleo & $3,1(, 8)$ & $3,1(, 5)$ & 1123,500 &, 448 \\
Orden publico & $3,4(, 6)$ & $3,1(, 6)$ & 920,500 &, 030 \\
Seguridad ciudadana & $3,3(1,3)$ & $3,3(1,3)$ & 1151,500 &, 574 \\
Hábitos culturales & $2,4(1,5)$ & $2,8(1,3)$ & 904,000 &, 022 \\
Aculturación & $3(1,6)$ & $3(1)$ & 1162,500 &, 628 \\
Infidelidad & $3,5(1,7)$ & $3,6(1,4)$ & 1145,500 &, 546 \\
\hline
\end{tabular}

Fuente: Autores

La comparación de variables de estudio según el nivel educativo mostró diferencia significativa en xenofobia, donde se observa una relación inversamente proporcional; es decir, a menor nivel educativo mayor nivel de xenofobia.

En cuanto a la percepción de oportunidad de empleo se identificó una diferencia significativa entre los grupos, mostrando que, a mayor nivel educativo mayor es la percepción de amenaza en cuanto al acceso al mercado laboral.

El puntaje obtenido en la subescala de hábitos indico que los participantes con el nivel educativo de Primaria mostraron una alta percepción negativa al respecto de los hábitos culturales de los migrantes venezolanos.

En cuanto a la aculturación, igualmente, se observó una diferencia significativa entre grupos, mostrando la mayor tendencia en la percepción 
negativa en los niveles educativos más bajos en comparación con las personas con mayor formación académica.

Por último, la variable infidelidad muestra el más alto puntaje en el grupo de participantes con nivel educativo de primaria, disminuyendo progresivamente con el avance en el nivel educativo.

Tabla N 3. Comparación de variables de estudio según nivel educativo

\begin{tabular}{lccccccc}
\hline & & & & & \\
& $3,9(2,2)$ & $2,5(1,0)$ & $2.1(, 7)$ & $2,1(1,3)$ & 18,304 &, 000 \\
\hline Xenofobia & $3(, 4)$ & $3(1)$ & $3,3(, 5)$ & $3,2(, 5)$ & 16,316 &, 001 \\
Oportunidad de empleo & & $3,1(, 4)$ & $3,6(, 7)$ & $3,3(, 8)$ & $3,1(1)$ & 6,367 &, 095 \\
Orden publico & $2,8(1,8)$ & $3,6(1)$ & $3,1(1,3)$ & $3(1)$ & 5,649 &, 130 \\
Seguridad ciudadana & $3,4(1,1)$ & $2,5(1)$ & $2,2(1,4)$ & $2,5(1)$ & 13,505 &, 004 \\
Hábitos culturales & $3,6(1,6)$ & $3,4(1,6)$ & $2,8(1,2)$ & $2,8(2,2)$ & 9,375 &, 025 \\
Aculturación & $4,6(1,6)$ & $3,7(2,3)$ & $3,5(1,8)$ & $3,0(1)$ & 17,820 &, 000 \\
\hline Infidelidad & & & & & & \\
\hline
\end{tabular}

Fuente: Autores

La comparación de variables según el estrato socioeconómico mostró diferencias significativas en orden público, mostrando una percepción más negativa en los estratos más bajos; y en la seguridad ciudadana, donde se evidencia una percepción de amenaza más alta a la seguridad personal de los ciudadanos en los estratos más altos.

Por último, la variable de infidelidad mostró puntajes más altos en los grupos de participantes pertenecientes a los estratos 1 y 2 , mostrando la tendencia a la disminución a medida que aumenta el estrato.

Sin embargo, estos resultados deben someterse a una nueva verificación mediante una muestra con mayor representatividad de los estratos más altos con el fin de evitar sesgos. 
Tabla N 4. Comparación de variables de estudio según estrato

\begin{tabular}{|c|c|c|c|c|c|c|c|}
\hline & 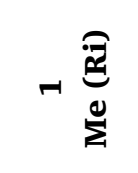 & 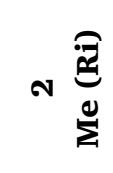 & ๓ & 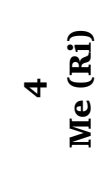 & 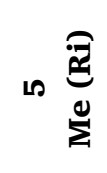 & 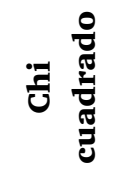 & $\frac{a}{\frac{0}{\pi}}$ \\
\hline Xenofobia & $2,8(2,6)$ & $2,5(1)$ & $2,2(1,8)$ & $3,5(0)$ & $3,5(0)$ & 3,080 & ,544 \\
\hline Oportunidad de empleo & $3(, 8)$ & $3,1(, 5)$ & $3(, 6)$ & $3,6(0)$ & $3,4(0)$ & 4,284 & ,369 \\
\hline Orden publico & $3,5(, 7)$ & $3,5(, 7)$ & $3,3(1)$ & $2,8(0)$ & $2,7(0)$ & 7,852 & ,047 \\
\hline Seguridad ciudadana & $2,8(1,7)$ & $2,7(1)$ & $2,8(, 4)$ & $3,3(0)$ & $3,3(0)$ & 14,323 & ,006 \\
\hline Hábitos culturales & $2,7(1,6)$ & $2,5(1)$ & $2,5(1,3)$ & $2,4(0)$ & $2,5(0)$ & 2,135 & ,711 \\
\hline Aculturación & $2,9(1,2)$ & $3,1(1)$ & $2,5(1,7)$ & $3,2(0)$ & $3,5(0)$ & 7,057 & 133 \\
\hline Infidelidad & $4(1,9)$ & $3,5(1)$ & $2,8(1,9)$ & $2,1(0)$ & $2(0)$ & 8,623 & 041 \\
\hline
\end{tabular}

Fuente: Autores

\section{Discusión}

En primer lugar, en resultados llama la atención un alto nivel de percepción social negativa al respecto de los migrantes venezolanos que manifestaron los participantes del estudio.

Es interesante que la variable de infidelidad obtuvo puntajes más altos, indicado la tendencia de habitantes de Sincelejo encuestados a considerar que los migrantes venezolanos son potencialmente culpables por los problemas de infidelidad en las parejas de residentes del lugar. En este aspecto mayor negatividad se expresa al respecto de mujeres venezolanas, consideradas como amenaza y tentación para la infidelidad masculina. Esta postura machista discriminatoria también es resaltada por el estudio de Malax et al. (2019), quienes identificaron en Perú, Colombia y Ecuador las actitudes de prejuicio y machismo hacia las mujeres venezolanas, donde la mayoría de los participantes expresaron que las mujeres venezolanas migrantes terminarían ejerciendo la prostitución.

Igualmente, se observó que a menor nivel educativo y menor estrato socioeconómico (estratos 1 y 2) los participantes mostraron una mayor percepción negativa del problema de infidelidad como consecuencia de migración venezolana. .

Los aspectos de seguridad ciudadana, orden público y oportunidades de empleo también mostraron altos niveles de percepción negativa por parte 
de los participantes, mostrando que los habitantes de Sincelejo perciben a los migrantes venezolanos como amenaza para la seguridad de la población residente. Estos resultados van en la misma línea que los hallazgos de Malax et al. (2019), que resaltan una percepción negativa sobre los migrantes venezolanos como causantes de inseguridad y delincuencia, al igual como amenaza para los empleos laborales de residentes y los problemas en el sistema de seguridad social.

$\mathrm{Al}$ respecto de lo anterior, las mujeres participantes del presente estudio mostraron una percepción significativamente más negativa que los hombres en cuanto a la consideración de los venezolanos como amenaza para el orden público de la comunidad. En este aspecto, algunos estudios han encontrado que las mujeres tienen un mayor nivel de intranquilidad o temor hacia la inseguridad que se presenta dentro de sociedad, mostrando una mayor preocupación constante de agresiones que afectan su integridad femenina como lo es riesgo a la violación y ataques físicos; esta situación configura los hábitos sociales de la población femenina, quienes consideran el espacio público como un lugar de riesgo para el desarrollos de sus actividades diarias y deciden abstenerse a la realización de su cotidianidad para evitar dichos riesgo (Madriz, 2001; Pain, 1995).

Por otro lado, los participantes de los estratos más bajos también perciben con mayor negatividad el efecto de migración venezolana en el orden público, relacionado con peleas y disturbios callejeros; y los participantes de los estratos más altos perciben con mayor negatividad el asunto de seguridad ciudadana, relacionada más con robos de viviendas, atracos a mano armada en la calle, etc.

Es importante resaltar estos datos obtenidos en el presente estudio y traer a colación las observaciones de Malax et al. (2019), quienes resaltan que en la mayoría de las situaciones las migraciones, incluyendo en este caso, la migración venezolana, solo acentúan o exasperan las problemáticas a nivel social o económico que ya estaban presentes con anterioridad en la comunidad receptora. Es evidente que, tanto los problemas de orden público en los estratos socioeconómicos más bajos, como la preocupación por la seguridad personal en los estratos más altos, ha sido un hecho que ha estado presente antes del inicio de la migración venezolana, formando parte de otras tantas problemáticas a nivel social presentes en a la sociedad colombiana. En este orden de ideas, es importante concientizar a la población receptora al respecto de las problemáticas presentes con el fin de evitar las generalizaciones y el aumento innecesario de percepciones y actitudes negativas frente a los migrantes venezolanos. 
En cuanto a la oportunidad de empleo se observó que los participantes con mayor nivel educativo de carrea universitaria y posgrados, expresaban mayor percepción negativa sobre los migrantes como amenaza para el empleo. Lo anterior puede deberse al hecho de que los residentes encuestados con mayor preparación académica perciben a los migrantes venezolanos con estudios superiores como amenaza de competencia en el sector educativo y empresarial. Estos hallazgos no concuerdan con lo afirmado por Jackson et al. (2001), quienes afirma que las personas con menores ingresos y menor preparación manifiestan actitudes más negativas hacia la inmigración, ya que deben competir con estos por salarios y trabajos menos calificados.

En este caso, al igual como el de orden público y seguridad ciudadana, es necesario considerar las condiciones preexistentes de empleo en la sociedad receptora, ya que una difícil situación económica puede aumentar la percepción negativa de los inmigrantes, tal como lo encontró en su estudio Berry (2001), demostrando que la actitud más negativa hacia la inmigración se presenta en situaciones económicas más difíciles y se vuelve más positiva cuando la economía mejora. Lo anterior lleva a plantear la necesidad de considerar hasta donde la precepción de amenaza de los migrantes para el empleo es más perceptual que real, siendo estos percibidos como consumidores de recursos de una comunidad como puestos de trabajo o de seguridad social, que de por sí ya son escasos.

Se observó un nivel medio alto de xenofobia en la muestra del estudio, siendo los hombres quienes mostraron niveles más altos que las mujeres. Los hallazgos del presente estudio están acordes con la investigación de Navas y Sánchez (2009), quienes revelan que las mujeres y personas que han relacionado con persona inmigrantes muestran actitudes más favorables hacia estos. En este aspecto, la actitud más favorable en el género femenino podría estar relacionado con lo plateado por Álvarez et al. (2020), quienes indican que las mujeres presentan mayor empatía, prosocialidad y menor agresividad que los hombres.

Igualmente, las personas con un menor nivel educativo mostraron mayor xenofobia que los participantes con mayores niveles educativos. Estos hallazgos concuerdan con lo planteado por un estudio realizado en la Unión Europea que indico que las personas con mayor nivel educativo tenían actitudes más favorables hacia los inmigrantes (IOE, 2003). Igualmente, algunos autores indican que las actitudes más xenófobas se dan con mayor intensidad en los estratos socioeconómicos más bajos, debido a que perciben a los inmigrantes como competidores en cuestión laboral y de servicios sociales, reclamando protección ante la precariedad y argumentando que los extranjeros aparecen 
como consumidores de escasos recursos disponibles (Díez-Nicolás y Ramírez, 2001; Solé et al., 2001).

Y, por último, los aspectos de aculturación y hábitos culturales mostraron nivel medio alto de percepción social negativa, mostrando un rechazo bastante alto por las costumbres, hábitos culturales o prácticas de los inmigrantes venezolanos y la tendencia a cambiar y dominar por la fuerza o la violencia a la cultura migrante. En este aspecto, los hombres mostraron una percepción más negativa de las costumbres y hábitos culturales de la población migrante, lo cual se relaciona, también, con más altos niveles de xenofobia, manifiesta por estos. Igualmente, los participantes de menor nivel educativo mostraron una percepción significativamente más negativa al respecto de hábitos culturales de los inmigrantes y una mayor tendencia de eliminar su cultura adaptando a los migrantes a la cultura colombiana.

Estos resultados representan una confirmación a los hallazgos expuestos en la investigación de Ramos De Olivera et al. (2005), que proponen que algunas culturas que presentan distancias a nivel jerárquico se enfocan en la promoción de valores tradicionales y en defensa de los estereotipos sociales generacionales, y que varían directamente en relación con los bajos niveles educativos en concordancia al rechazo y actitudes poco receptivas hacia la población migrante.

En este aspecto, algunos autores también indican que lo negativo hacia los migrantes surge desde la calidad de vida que presentan las personas de las comunidades receptoras, ya que, entre más precarias son las condiciones económicas y educativas, mayores son los prejuicios hacia esta población, expresándose estos tanto en los escenarios sociales y laborales, como a nivel individual y colectivo (Ramos De Olivera et al., 2005).

Igualmente, en muchas sociedades se manifiestan actitudes de rechazo a otras culturas y tendencia de asimilarlas y adaptarlas por la fuerza a las culturas predominantes, fenómeno relacionado con la identidad cultural según el concepto de la "normalidad", donde todo lo diferente y ajeno se considera como no legítimo y se reclama su aislamiento y asimilación como un elemento anómalo (IOE, 2003). En este sentido, los estudios indican que un buen nivel de patriotismo que es comprensivo y no agresivo y una conciencia sobre el orgullo nacional puede ser factor que promueve aceptación de inmigración; sin embargo, un fuerte nacionalismo sumado a una estima colectiva alta e insegura, actúan como factor facilitador de rechazo hacia lo inmigrantes inmigración (Páez et al., 2004). 
A modo de conclusión se puede afirmar que concientizar a las poblaciones frente al fenómeno de migración, tal como plantean Malax et al. (2019), creando una percepción de ésta no como un problema económico, social y cultural para las comunidades receptoras, sino como una oportunidad de construir sociedades más incluyentes y tolerantes, permite minimizar las consecuencias sociales como el rechazo, la estigmatización y las conductas con iniciativas xenofóbicas que dañan la integridad de la comunidad en general. La imagen negativa a la que muchos migrantes deben hacer frente, facilita la agresión, la humillación y los prejuicios culturales que limitan su acceso a las oportunidades para mejorar su calidad de vida, por tanto, sigue siendo una población que no está exenta a la opresión y al cuestionamiento social.

Por último, es necesario resaltar la debilidad del presente estudio en cuanto al reducido tamaño de la muestra, siendo necesario su ampliación en futuros estudios. Otra limitación que debe considerarse es el instrumento empleado en el estudio, que, aunque cuenta con la validez de contenido y un alfa Cronbach alto, seria importante realizar una más detallada valoración de sus propiedades psicométricas para futuros estudios. Igualmente, es necesario tener en cuenta el alto porcentaje de los participantes de los estratos socioeconómi$\cos 1$ y 2 en la muestra del estudio, lo cual podría generar sesgo en algunos resultados, planteando la necesidad de replicar el estudio en muestras mas representativas en relación a otros estratos.

\section{Referencias}

ACNUR (La Agencia de la ONU para los refugiados) (2017). Tendencias globales, Desplazamiento forzado en 2017. https://www.acnur.org/5b2956a04.pdf

Alvarez, P., Carrasco, M., y Fustos, J. (2010). Relación de la empatía y género en la conducta prosocial y agresiva, en adolescentes de distintos tipos de establecimientos educacionales. Revista Iberoameicana de psicología: Ciencia y tecnología, 3(2), 2736. https://doi.org/10.33881/2027-1786.rip.3203

Bermúdez, Y., Mazuera-Arias, R., Albornoz-Arias, N., Morffe, M. (2018). Informe sobre la movilidad humana venezolana. Realidades y perspectivas de quienes emigran. Venezuela: Servicio Jesuita a Refugiados. https://drive.google.com/file/d/11OMt NWuHeWjb3ykaerahBFPOBSvdsx5F/view

Berry, J. (2001). A psychology of immigration. Journal of Social Issues, 57(3), 615631. https://doi.org/10.1111/0022-4537.00231

Cea D’Ancona, M. (2005). La exteriorización de la xenofobia. Revista Española de Investigaciones Sociológicas, 112, 197-230, http://www.reis.cis.es/REIS/jsp/ 
REIS.jsp?opcion $=$ articulo\&ktitulo $=2015$ \&autor $=$ M.\%AA+\%C1NGELES +CEA+D\%27ANCONA

Consejo Nacional de Población, México (2010). Índices de intensidad migratoria México-Estados Unidos 2010. http://www.conapo.gob.mx/work/models/ CONAPO/intensidad_migratoria/pdf/Efectos.pd

Departamento de Asuntos Económicos y Sociales (DAES, ONU). (2016). International Migration Report, 2015. https://www.un.org/en/development/desa/population/ migration/publications/migrationreport/docs/MigrationReport2015_Highlights.pdf

Díez-Nicolás, J., y Ramírez, M. (2001). Inmigración en España, Una década de investigaciones. Madrid: IMSERSO

El Espectador (01 de Agosto de 2019). Migrantes venezolanos en Colombia según migración colombiana. https://www.elespectador.com/noticias/el-mundo/ hay-1408055-migrantes-venezolanos-en-colombia-segun-migracion-colombiaarticulo-873955

IOE (2003). Inmigración y Ciudadanía: España en el contexto de las migraciones internacionales. Madrid: Fundamentos.

Jackson, J., Brown, K., Brown, N., y Marks, B. (2001). Contemporary immigration policy orientations among dominant-group members in Western Europe. Journal of Social Issues, 57 (3), 431-456. https://doi.org/10.1111/0022-4537.00222

González, M. (2011). Los efectos de las migraciones internacionales en el contexto de la globalización. En F. J. García Castaño y N. Kressova. (Coords.) . Actas del I Congreso Internacional sobre Migraciones en Andalucía. (pp. 2033-2040). Granada: Instituto de Migraciones. https://dialnet.unirioja.es/servlet/articulo? codigo $=4050052$

Organización Internacional para las migraciones (OIM) (2010). Informe sobre las Migraciones en el mundo 2010. https://publications.iom.int/system/files/pdf/ wmr_2010_spanish.pdf

Organización Internacional para las migraciones (OIM) (2018). Informe sobre las Migraciones en el mundo 2018. https://publications.iom.int/system/files/pdf/ wmr_2018_sp.pdf

Madriz, E. (2001). A las niñas buenas no les pasa nada malo. México: Siglo XXI.

Malax, A., Díez, L., Rivero, p., Santamaría, C. (2019). Sí, pero no aquí, percepciones de xenofobia y discriminación hacia migrantes de Venezuela en Colombia, Ecuador y Perú. Informe de investigación de OXFAM, https://oxfamilibrary.openrepository. com/bitstream/handle/10546/620890/bp-si-pero-no-aqui-251019-es. pdf sequence $=8 \&$ isAllowed $=\mathrm{y}$ 
Ministerio y salud y protección social. (s.f.). Plan de respuesta del sector de salud al fenómeno migratorio. https://www.minsalud.gov.co/sites/rid/Lists/ BibliotecaDigital/RIDE/DE/COM/plan-respuesta-salud-migrantes.pdf

Navas, L., y Sánchez, A. (2009). Actitudes hacia la inmigración de los estudiantes de psicología chilenos: análisis diferenciales. SUMMA Psicológica, 6 (2), 119 - 130, https://doi.org/10.18774/summa-vol6.num2-67

Lara, A. (2007). Migraciones internacionales, seguridad y xenofobia: los límites del modelo francés de integración. Oasis. 12, 209-227, https://revistas.uexternado. edu.co/index.php/oasis/article/view/2419

Páez, D., Fernández, I., Ubillos, S., y Zubieta, E. (2004). Psicología Social, Cultura y Educación. Madrid: Ed. Pearson Educación.

Pain, R. (1995). Elderly women and fear of violent crime: The least likely victims? A reconstruction of the extent and nature of risk. British Journal of Criminology 35 (4), 584-598, https://doi.org/10.1093/oxfordjournals.bjc.a048548

Solé, C. Parella, S., Alarcón, A., Bergalli, V. (2001). El impacto de la inmigración en la sociedad receptora. Reis, 90(7), 131-157, http://www.reis.cis.es/REIS/PDF/ REIS_090_07.pdf

Suárez, D. (2008). Causas y efectos de la migración internacional. Perspectivas, (22), 161-180, https://www.redalyc.org/articulo.oa?id=4259/425942158006

Ramos De Olivera, D., Techio, E. M., Paez, D., y Herranz, C. (2005). Factores predictores de las actitudes ante la inmigración. Revista de Psicología Social 20(1):19-37, https://doi.org/10.1174/0213474052871060

Ripoll, S., Navas-Alemán, L., Arias, M., Larsen, D., Vasquez, J., Cordero, B., Ruiz, E., Manzano, D., Vasquez, G., Tamayo, C., Delgado, A., Salazar, R., Rapido, I., Zepeda, B., Mouly, C., Córdova, D., Texido, I., Sánchez, C. (2018). Xenofobia y discriminación hacia refugiados y migrantes venezolanos en Ecuador y lecciones aprendidas para la promoción de la inclusión social. Social Science in Humanitarian Action plataform, https://www.socialscienceinaction.org/

Velarde, M. (2019). Representación mediática de la dicotomía xenofobia-endofobia de la migración venezolana en el Perú del año 2017 al 2018. Tesis de grado Académico de Bachiller en Comunicaciones. Universidad San Ignacio de Loyola, http:// repositorio.usil.edu.pe/bitstream/USIL/9179/1/2019_Velarde-Ramirez.pdf 